\title{
Is the current concept of recurrent ovarian carcinoma as a chronic disease also applicable in platinum resistant patients?
}

\author{
Uwe Güth · Dorothy Jane Huang • \\ Andreas Schötzau $\cdot$ Edward Wight
}

Received: 4 April 2009 / Accepted: 9 June 2009 / Published online: 24 June 2009

(C) Springer-Verlag 2009

\begin{abstract}
Purpose The treatment of recurrent ovarian carcinoma (ROC) has become increasingly oriented according to the therapy principles of a chronic disease. We evaluated whether it is justifiable to also apply this concept to the treatment of platinum resistant patients with their known poor prognosis and short overall survival (OS).

Methods We analyzed the overall courses of 85 unselected ROC patients and defined the following groups: A, platinum resistant patients $(n=39)$; subgroup A.1, those who received no or at maximum one line of palliative chemotherapy $(n=15,38.5 \%)$; subgroup A.2, those who received $\geq$ two therapy lines $(n=24,61.5 \%)$; B, platinum sensitive patients, $n=46$.

Results Group A had significantly lower OS than group B (median: 16 vs. 25 months; $p=0.019$ ). Group A.1 had significantly worse outcome compared to group A.2 (median: 5 vs. 21.5 months; $p<0.001$ ). The comparison between study group A.2 and group B showed comparable survival rates $(p=0.738)$. Considering only the patients who had completed treatment courses, the median number of therapy lines administered was higher in group A.2 than in group B (4 vs. $3 ; p=0.008$ ).

Conclusions There is not only the known dichotomy between platinum sensitive and resistant ROC patients, but
\end{abstract}

U. Güth $(\bowtie) \cdot$ D. J. Huang $\cdot$ E. Wight

Department of Gynecology and Obstetrics,

University Hospital Basel (UHB), Spitalstrasse 21,

4031 Basel, Switzerland

e-mail: ugueth@uhbs.ch

A. Schötzau

Schötzau and Simmen Institute for Biomathematics,

Malzgasse 9, 4052 Basel, Switzerland rather also within the platinum resistant subgroup itself. There is a considerably large subgroup of platinum resistant patients who will subsequently enter a phase where multiple treatment programs will be considered and administered. These patients have similar survival rates compared to those from the platinum sensitive patient group and the therapy principles of a chronic disease are applicable.

Keywords Ovarian carcinoma - Recurrent disease · Chronic disease $\cdot$ Platinum-resistance $\cdot$ Chemotherapy Outcome

\section{Introduction}

The majority of women with ovarian carcinoma are diagnosed with advanced-stage disease. Despite continuing improvements in first-line treatment (the current standard is platinum-based chemotherapy regimens), most patients relapse and die of the disease within 5 years [1]. Currently, second-line therapy hardly provides a realistic chance of a cure; thus, the goals of treatment are different from first-line chemotherapy and include prolonging survival, improving cancer-related symptoms, delaying the development of symptoms, and improving quality of life [2].

Patients with recurrent ovarian carcinoma (ROC) are generally categorized into two primary groups: platinumsensitive, in which the disease recurs after 6 months from the time of first-line platinum-based chemotherapy, and platinum resistant, in which the progression-free survival (PFS) is less than 6 months from completion of treatment. This well-established dichotomy reflects the expected efficacy of further palliative systemic therapy options. While platinum sensitive patients experience response rates 
generally $>50 \%$ and have overall survival (OS) of $>1$ year, platinum resistant patients have demonstrated response rates of usually $<20 \%$ and an OS of $<12$ months [3-5].

In the last decade, ROC has become increasingly viewed as a chronic disease process $[2,6,7]$; the choice of systemic palliative therapy options takes this concept increasingly into account [8]. The goal of this study was to demonstrate whether it is justifiable to also apply this concept to the treatment of platinum resistant patients with their known poor prognosis and short OS. In order to address this issue, we analyzed the overall courses of an unselected population-based study cohort of patients whose ROC was diagnosed during a 10 year period (1997-2006).

\section{Patients and methods}

The Basel Ovarian Carcinoma Database is comprised of extensive data concerning clinical, histo- and pathomorphologic features and treatment characteristics of all patients whose primary epithelial ovarian cancer was diagnosed in the canton Basel-Stadt (Basel, Switzerland) since 1990. For this study, we considered the data from patients whose ROC was initially diagnosed from 1997 to 2006 and who were treated postoperatively with at least three cycles of platinum-based chemotherapy as first-line treatment (median number of cycles: 6, range 3-11). Since the use of palliative chemotherapy options was a crucial factor evaluated in this study, we did not include patients for whom the choice of possible treatment options were limited by advanced age (older than 75 years).

Ultimately, 85 patients were analyzed in this study. The clinicopathologic, treatment and outcome characteristics of these patients are summarized in Tables 1 and 2. Most of the patients had FIGO (International Federation of Gynecology and Obstetrics) stages I-III at initial diagnosis $(n=77)$. In the remaining eight patients who were classified as having stage IV disease, the grouping into this stage was based only on the diagnosis of pleural involvement and/or liver parenchymal metastases; patients with other distant metastases (lesions beyond the abdominal cavity) were excluded from analysis. Of the entire study cohort, 39 patients had a PFS $<6$ months after completion of first-line chemotherapy and were therefore considered as being platinum resistant (study group A). 46 patients who were platinum sensitive (PFS $>6$ months) comprised study group B.

No patients were lost to follow-up and we could provide complete information regarding palliative therapy course (number of chemotherapy lines and the agents administered) and outcome for all patients. Information concerning palliative treatment was obtained from 11 oncologic units in Switzerland, as well as neighboring regions in Germany and France. The patients were followed until death or, if they remained alive and disease free, for a minimum of 24 months (conclusion of the data collection in July 2008). Overall survival was defined as the interval from the date of diagnosis of ROC to the date of death. In this manner, the recurrent disease-specific survival was calculated.

One patient in study group B had isolated brain metastases, i.e., no recurrent disease in the abdominal region, and received only radiotherapy. All other patients included in the study had recurrence in abdominal sites as a manifestation of recurrent disease. The majority of the patients died of ROC (group A: $n=38,97.4 \%$; group B: $n=40,87.0 \%$ ). One patient in group A and six patients in group B were alive at the conclusion of the observation period; four patients in group B, as well as the one patient in group A, had ongoing palliative therapies. In two further patients in group B, localized recurrent disease was diagnosed. In one case, a 47-year-old woman had a recurrent tumor mass in the pelvis, which was surgically removed (no postoperative residual disease), and postoperatively received a secondline chemotherapy with six cycles of carboplatin and paclitaxel. The other patient (age, 74 years) had a histologically proven recurrence as a fixed pelvic mass eroding the vaginal mucosa. This 74-year-old patient was treated with six cycles of carboplatin as monotherapy. These two patients have experienced a long asymptomatic period (49 and 55 months, respectively) up until the conclusion of the observation period and could be even considered as potentially cured.

It was the goal of our study to evaluate whether the therapy principles of a chronic disease can also be applied in platinum resistant patients. An important feature of a chronic disease therapy situation is that, besides demonstrating a long enough survival time to be considered as chronic, the ability to administer antineoplastic therapy at all needs to be possible. Therefore, we further subdivided the study group of the platinum resistant patients as follows:

Group A.1 patients who received no antineoplastic systemic treatment or at maximum one line of palliative chemotherapy, $n=15(38.5 \%)$.

Group A.2 patients who received $\geq$ two therapy lines, $n=24(61.5 \%)$.

The study design and data collection methods were approved by our institutional review board.

\section{Statistical analysis}

To predict the OS with ROC, we used the Kaplan-Meier method. Patients who were alive at the conclusion of the observation period were censored in the statistical analyses. Statistical differences between groups in terms of survival curves were analyzed using the log rank test. To 
Table 1 Clinicopathologic and outcome characteristics of 85 patients with recurrent ovarian carcinoma first diagnosed 1997-2006
Group A, platinum resistant patients $(n=39)$; Group B, platinum sensitive patients $(n=46)$

$C T$ chemotherapy, FIGO International Federation of Gynecology and Obstetrics

\begin{tabular}{|c|c|c|}
\hline Variable & Group A & Group B \\
\hline Entire group, $n(\%)$ & $39(100)$ & $46(100)$ \\
\hline \multicolumn{3}{|l|}{ FIGO stage at initial diagnosis } \\
\hline Stage I & $3(7.7)$ & $2(4.3)$ \\
\hline Stage II & $3(7.7)$ & $6(13.1)$ \\
\hline Stage III & $27(69.2)$ & $36(78.3)$ \\
\hline Stage IV & $6(15.4)$ & $2(4.3)$ \\
\hline \multicolumn{3}{|l|}{ Histologic subtype } \\
\hline Serous & $29(74.4)$ & $41(89.1)$ \\
\hline Mucinous & $4(10.2)$ & - \\
\hline Endometriod & $3(7.7)$ & $4(8.7)$ \\
\hline Clear-cell & $3(7.7)$ & $1(2.2)$ \\
\hline \multicolumn{3}{|l|}{ Residual disease after cytoreductive surgery } \\
\hline No macroscopic residual disease & $10(25.6)$ & $26(56.5)$ \\
\hline Residual disease $<1 \mathrm{~cm}$ & $9(23.1)$ & $10(21.7)$ \\
\hline Residual disease $1-2 \mathrm{~cm}$ & $9(23.1)$ & $6(13.1)$ \\
\hline Residual disease $>2 \mathrm{~cm}$ & $10(25.6)$ & $4(8.7)$ \\
\hline Unknown amount of residual disease & $1(2.5)$ & - \\
\hline \multicolumn{3}{|l|}{ Postoperative platinum-based CT } \\
\hline Platinum monotherapy & $9(23.1)$ & $8(17.4)$ \\
\hline Platinum + taxane & $23(59.0)$ & $25(54.3)$ \\
\hline Platinum + cyclophosphamide & $6(15.4)$ & $12(26.1)$ \\
\hline Platinum + other CT agent & $1(2.5)$ & $1(2.2)$ \\
\hline \multicolumn{3}{|l|}{ Age at diagnosis of recurrent disease } \\
\hline Mean (range) & 57.3 years $(29-75)$ & 59.2 years $(33-75)$ \\
\hline \multicolumn{3}{|l|}{ Outcome status } \\
\hline Died of ovarian cancer & $38(97.4)$ & $40(87.0)$ \\
\hline Died of other causes & - & - \\
\hline Alive (ongoing therapy with evident disease) & $1(2.6)$ & $4(8.7)$ \\
\hline Alive (no evidence of disease $>48$ months) & - & $2(4.3)$ \\
\hline
\end{tabular}

Table 2 Number of therapy lines received by patients who died of recurrent ovarian carcinoma

\begin{tabular}{lll}
\hline & Group A & Group B \\
\hline Total no. of patients, $n(\%)$ & $38(100)$ & $40(100)$ \\
No systemic therapy & $5(13.2)$ & $2(5.0)$ \\
1 therapy line & $10(26.3)$ & $5(12.5)$ \\
2 therapy lines & $4(10.5)$ & $12(30.0)$ \\
3 therapy lines & $6(15.8)$ & $9(22.5)$ \\
4 therapy lines & $7(18.4)$ & $7(17.5)$ \\
5 therapy lines & $3(7.9)$ & $3(7.5)$ \\
6 therapy lines & $2(5.3)$ & $1(2.5)$ \\
7 therapy lines & - & $1(2.5)$ \\
9 therapy lines & $1(2.6)$ & - \\
\hline
\end{tabular}

Group A, platinum resistant patients $(n=38)$; Group B, platinum sensitive patients $(n=40)$

compare ordinal variables (number of therapy lines) between two groups, the nonparametric exact Wilcoxon test was performed.
To predict how the platinum resistant patients fell into the subgroups A.1 or A.2, multiple logistic regression was performed. Independent predictors were age, relapse-free period, and the amount of residual disease after cytoreductive surgery.

A $p$ value $<0.05$ was considered to be significant. Odds ratio (OR) and the corresponding $95 \%$ confidence interval (CI) were reported. For continuous variables, ORs were calculated as the ratio from the third to the first quantile of the corresponding distribution. Statistical analyses were performed with R Development Core Team software, version 2.7.0 (Vienna, Austria).

\section{Results}

A comparison between both study groups showed that group A had significantly lower OS than platinum sensitive patients (Fig. 1; $p=0.019$ ). The 1-year adjusted survival rate was $53.9 \%$ for group A compared to $82.6 \%$ for group 


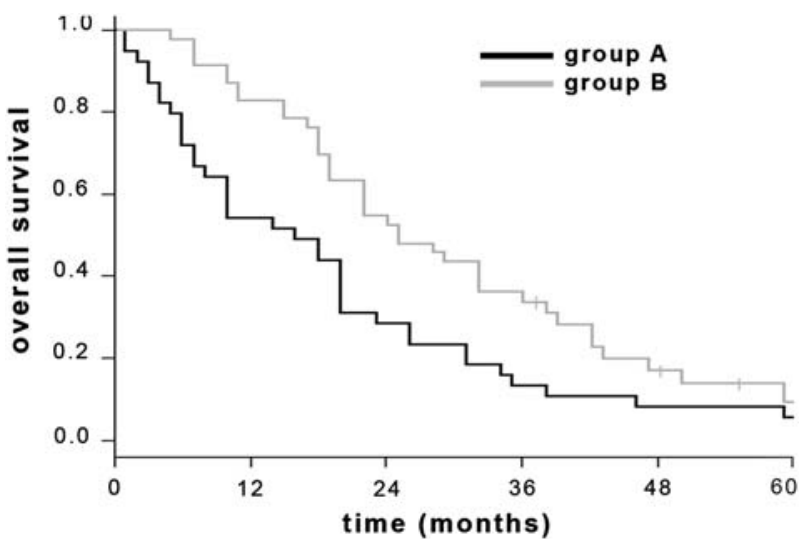

Fig. 1 Overall survival among 85 patients with recurrent ovarian carcinoma. Group A, platinum resistant patients $(n=39)$; Group B, platinum sensitive patients $(n=46)$

$\mathrm{B}$; the 3-year rates were 12.8 and $33.5 \%$, and the 5-year rates were 5.1 and $8.9 \%$, respectively. The median OS time was 16 months in group A and 25 months in group B.

If one subdivides the study group with platinum resistant patients further according to the number of therapy lines administered in the palliative setting, one finds again a clear dichotomy. Patients who received no or maximal one therapy line (group A.1) have significantly worse outcome compared to group A.2, which was comprised of patients who received $\geq 2$ therapy lines (Fig. $2 ; p<0.001$ ). In group A.1, no patient survived longer than 10 months. In group A.2, the 1-year adjusted survival rate was $87.5 \%$, while the 3 -year rate was $20.8 \%$ and the 5 -year rate was $8.3 \%$. The median OS time was 5 months in group A.1 and 21.5 months in group A.2. In a multivariate analysis of the patients of study group A, a short relapse free period was a

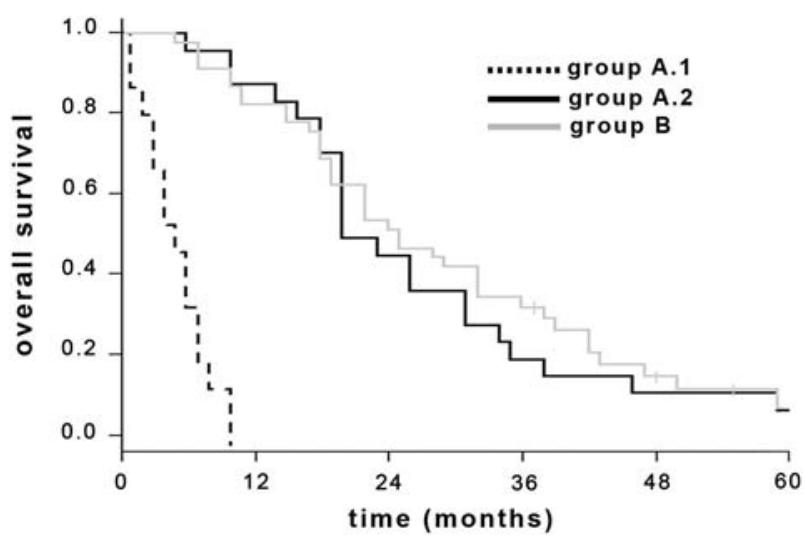

Fig. 2 Overall survival among 85 patients with recurrent ovarian carcinoma. Group A.1, platinum resistant patients who received no second-line chemotherapy or at maximum one line of palliative treatment $(n=15)$; Group A.2, platinum resistant patients who received $\geq 2$ palliative chemotherapy lines $(n=24)$; Group B, platinum sensitive patients $(n=46)$ significant factor for the patients that eventually fell into sub-study group A.1 (OR, 5.75; 95\% CI, 1.12-29.54; $p=0.036)$. Age (OR, 0.39; 95\% CI, 0.12-1.26; $p=0.11$ ) and residual disease after cytoreductive surgery (OR, 4.26; 95\% CI, $0.68-26.63 ; p=0.12$ ) were shown not to be significant variables.

The comparison between study group A.2 and platinum sensitive patients showed comparable survival rates (Fig. 2, $p=0.738$ ). Considering only the patients who ultimately died of their disease, i.e., who had completed treatment courses, the median number of therapy lines administered was higher in the platinum resistant subgroup than in the platinum sensitive one (group A.2: 4 lines vs. group B: 3 lines; $p=0.008$ ).

\section{Discussion}

In the last decade, ROC has become increasingly viewed as a chronic disease process [2, 6-8]. Chronic diseases are by definition long-lasting or recurrent and require a long period of treatment, supervision, observation or care; they are caused by nonreversible pathological alterations, leave residual disability, and can be altered but not be cured by various therapies $[9,10]$. In any case, one main therapeutic goal in the management of chronic diseases, which can also be applied to the treatment of ROC, is fulfilled when a disease which requires periodic chemotherapy to control progressive course and symptoms can be treated with a strategy that permits stabilization with treatment regimens that have limited cumulative toxicity $[2,8]$.

Undoubtedly, through the introduction of a new generation of cytotoxic agents with safer profiles (above all, liposomal doxorubicin, gemcitabine and topotecan) and of course, through considerable advances in supportive care, the therapy concepts of chronic disease can be better implemented today compared to earlier times. We deliberately chose our study period to ensure that the above mentioned new drugs were available on the market. In a recent study, we could demonstrate that oncologists, using the currently available agents, are increasingly able to accordingly adjust the therapies in ROC patients with this principle in mind (e.g., administration of significantly fewer combination therapies with more favorable toxicity profiles) [8].

There is no generally accepted definition as to how long a disease must last in order to be considered as chronic. In the case of rapidly progressive disease which leads to death within a few months, this is surely not justified. Therefore, it must be questioned whether the term chronic disease is appropriate in association with platinum resistant ROC. Despite numerous chemotherapeutic strategies (overview in [11]), the poor prognosis has remained unchanged for these patients. Rocconi et al. [12] evaluated the cost-effectiveness 
of palliative chemotherapy for platinum-resistant ROC and provocatively demonstrated that best supportive care was the only cost-effective strategy for these patients.

Our study demonstrates that there is not only a dichotomy between platinum sensitive and resistant ROC patients, but rather also within the platinum resistant subgroup itself. Some of the patients experienced a rapidly progressive course of disease. These patients quickly showed that they do not respond well to further chemotherapy or reject further treatment, due to shock and disappointment with the recently completed ineffective first-line therapy and its toxicities. For these patients, who have a median survival less than 6 months, the term chronic disease is surely not appropriate. On other hand, there is a considerably large subgroup of platinum resistant patients who will subsequently enter a phase where multiple treatment programs will be considered and administered. These patients have similar survival rates compared to those from the platinum sensitive ROC patient group. In these patients, as demonstrated above, the therapy principles of a chronic disease are applicable.

In general, we think that in platinum resistant ROC patients, therapeutic nihilism is not justified. The substantial percentage of patients with ovarian cancer who wish to continue active anticancer treatment despite the development of platinum- and taxane-resistant disease should be encouraged by their oncologists to begin or to continue second line therapies. The choice of treatment options will depend on any given patient's clinical situation and her ability and readiness to tolerate the known side effects of the selected drug.

Many authors have suggested that, from a clinical perspective, the definitions of platinum sensitivity and resistance may be arbitrary [2, 13, 14]. The principle of second-line therapy is that if the treatment is successful, patients will eventually have another recurrence and undergo additional rounds of therapy. In this sense, we also think that platinum-sensitive and resistant diseases are not necessarily different entities in terms of the concept of a chronic disease.

The limitations of our study, however, must be considered. First, our study comes from a single region of a small country with a high socioeconomic status. All inhabitants of Switzerland have universal access to health care and free access to all prescribed and approved drugs. These facts must be considered while interpreting our results. Secondly, our study analyzes retrospective data. One has to realize, however, that prospective randomized trials are unsuitable for addressing the topic of our study. There exists a vast amount of literature concerning the systemic therapy of ROC (overview in [1, 2, 7, 13-16], particularly for platinum resistant disease in [11]). However, nearly all clinical trials concentrate only on the feasibility and impact of defined therapy options, usually in second line treatment situations early after the initial diagnosis of ROC. They focus on the evaluation and comparison of particular antineoplastic agents and drugs, but in doing so, can only evaluate particular therapy options in pre-selected groups of patients in certain situations. Thus, their ability to describe the overall course of recurrent disease is limited. Only a few trials have evaluated the clinical efficacy of third-, fourth- or fifth-line therapies [17]; due to the heterogeneous composition of these study groups of heavily pretreated women, insights from therapy over the entire disease course is often only interpretable in a limited manner. In order to evaluate the treatment course of a chronic disease, however, it is essential to give an overview of the entire course of the disease. In our opinion, this is only achievable through the analysis of retrospective data of a populationbased cohort, as done in our study.

Conflict of interest statement The authors declare that they have no conflict of interest.

\section{References}

1. Ozols R, Rubin S, Thomas G, Robboy S (2005) Epithelial ovarian cancer. In: Hoskins W, Perez C, Young R (eds) Principles and practice of gynecologic oncology. Lippincott Williams \& Wilkins, Philadelphia, pp 895-987

2. Bukowski RM, Ozols RF, Markman M (2007) The management of recurrent ovarian cancer. Semin Oncol 34:S1-S15

3. Eisenhauer EA, Vermorken JB, van Glabbeke M (1997) Predictors of response to subsequent chemotherapy in platinum pretreated ovarian cancer: a multivariate analysis of 704 patients [see comments]. Ann Oncol 8:963-968

4. Ozols RF (1997) Treatment of recurrent ovarian cancer: increasing options-"recurrent" results. J Clin Oncol 15:2177-2180

5. Parmar MK, Ledermann JA, Colombo N et al (2003) Paclitaxel plus platinum-based chemotherapy versus conventional platinumbased chemotherapy in women with relapsed ovarian cancer: the ICON4/AGO-OVAR-2.2 trial. Lancet 361:2099-2106

6. Markman M (2006) Unresolved issues in the chemotherapeutic management of gynecologic malignancies. Semin Oncol 33:S33S38

7. Michener CM, Belinson JL (2005) Modern management of recurrent ovarian carcinoma. A systematic approach to a chronic disease. Oncology (Williston Park) 19:1277-1285 discussion 1285, 1288,1293

8. Güth U, Kann SR, Huang DJ et al (2009) Systemic therapy developments and their effects regarding the current concept of recurrent ovarian carcinoma as a chronic disease. Arch Gynecol Obstet [Epub ahead of print]

9. Lubkin I, Larsen P (2002) Chronic illness. Jones \& Bartlett, Sudbury

10. Norris S, Glasgow R, Engelgau M et al (2003) Chronic disease management: a definition and systematic approach to component interventions. Dis Manag Health Outcomes 11:477-488

11. Pectasides D, Psyrri A, Pectasides M, Economopoulos T (2006) Optimal therapy for platinum-resistant recurrent ovarian cancer: doxorubicin, gemcitabine or topotecan? Expert Opin Pharmacother 7:975-987 
12. Rocconi RP, Case AS, Straughn JM Jr et al (2006) Role of chemotherapy for patients with recurrent platinum-resistant advanced epithelial ovarian cancer: a cost-effectiveness analysis. Cancer 107:536-543

13. Herzog TJ (2006) The current treatment of recurrent ovarian cancer. Curr Oncol Rep 8:448-454

14. Modesitt SC, Jazaeri AA (2007) Recurrent epithelial ovarian cancer: pharmacotherapy and novel therapeutics. Expert Opin Pharmacother 8:2293-2305
15. Fung-Kee-Fung M, Oliver T, Elit L et al (2007) Optimal chemotherapy treatment for women with recurrent ovarian cancer. Curr Oncol 14:195-208

16. Ozols RF (2006) Challenges for chemotherapy in ovarian cancer. Ann Oncol 17(Suppl 5):v181-v187

17. Markman M (2001) Why study third-, fourth-, fifth-line chemotherapy of ovarian cancer? Gynecol Oncol 83:449-450 